\title{
Multibeam Bathymetric Data Processing and Applications Based on
}

\author{
Cygwin and MB-System \\ Xiao Chunqiao ${ }^{1}$,Zhang Wang $^{1}$, Lv Zhiwei $^{1}$ \\ ${ }^{1}$ Beihai Marine Engineering Prospecting Institute of State Oceanic Administration ,Shandong \\ Qingdao 266061,China
}

Keywords: Mb-System; Cygwin;Caris; Reson 7125

\begin{abstract}
Cygwin is a Unix-simulated environment running on the Windows platform.Mb-System is a Unix-based multi-beam bathymetric data process open source software that can be installed and run on Cygwin. This paper introduces the installation of MB-System on Cygwin and its main functions, and then the detailed processing of multibeam bathymetric data using MB-System is presented using data obtained from a dock in Qingdao. Finally, the results is compared with the one achieved from a commercial software to validate the reliability of MB-System. As MB-System is an open source software, it will be a nice tool to study multibeam principles and related data.
\end{abstract}

\section{Introduction}

Cygwin is a Unix-simulated environment running on the Windows platform, and it is developed by Cygnus Solutions, which has been acquired by Red Hat ${ }^{[1]}$. MB-Systemis an open source software for processing multibeam bathymetric data, which is funded by Monterey Bay Aquarium Research Institute and Lamont-Doherty Earth Observatory ${ }^{[2]}$. The code is distributed under the GNU/GPL license, and can be compiled and run on Darwin, Linux, Cygwin, Sun systems. The current version is 5.4.2209 ${ }^{[1,3]}$. Using Cygwin, MB-System can be run on Windows platform, so it can take full advantages of user-friendly GUI of Windows OS and the stability and flexibility of Unix OS.

Currently, widely used multibeam bathymetric data processing software in China are Caris Hips and Sips and Triton Perspective. Compared to these two commercial software, few people pay attention to and take advantage of MB-System, and we only find two related papers ${ }^{[3,4]}$. Compared to commercial software, getting started with MB-System might be relatively difficult,but it make us close to the data and help us understand the principles of multibeam and data acquisition and process by learning and manipulating MB-System software.

Firstly, the main functions of MB-System are introduced, and then the detailed processing of multibeam bathymetric data using MB-System is presented using data obtained from a dock in Qingdao, which is acquired using Reson SeaBat 7125 multibeam system. Finally, the results is compared with the one achieved from Caris to validate the reliability of MB-System.

\section{Installation and main functions of MB-System}

Download the latest MB-System zip file, compile and install it, then the executable programs will be under the bin directory ${ }^{[3,4,5]}$. The executable programs are functions achieved in MB-System, and can be divided into the following three categories:

(1) MBIO I/O library. It supports typical Multibeam Echo Sounders data format. The data format used in this paper is GSF (Generic Sensor Format) ${ }^{[6]}$. Related commands are mb7kpreprocess.exe, mbhysweeppreprocess.exe, mbswplspreprocess.exe, mbkongsbergpreprocess.exe, etc.;

(2) Data View. Related commands : 
mbdatalist, mbinfo,mbm_plot, etc.;

(3) Data Correction. Related commands :

mbedit,mbnavedit,mbvelocitytool,mbrollbias, etc.;

(4) Data Processing and Mapping. Related commands: mbset,mbprocess, mbm_grid, etc.

\section{Data Processing}

For the convenience of data processing, the acquired data were converted to MB-system data format from GSF format. Firstly, using mbinfo command to check the file information and format number, then do the batch conversion. The command is:

\$ls -1 | perl multi-copy.pl

Ls and perl are the command. The former is to list all the files in the directory. The latter is to execute multicopy.pl script file. The content of multicopy.pl is as following:

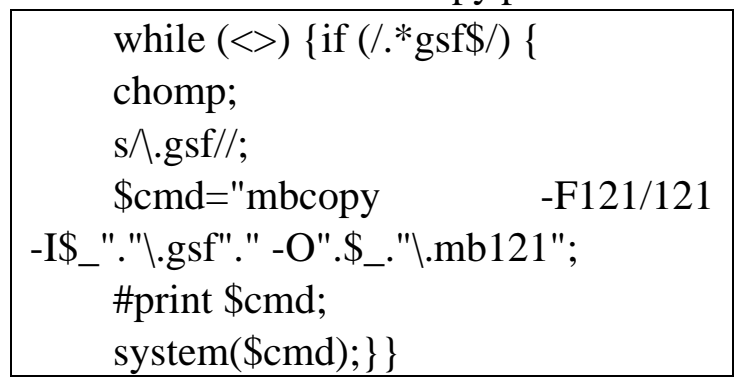

This script file is to convert all the .gsf files in the current directory to mbm121 data format. 121 means the format number of gsf format in MB-System.

After the format conversion, the mbdatalist can be used to the list of data files and generate three additional files, information statistics file (.inf), fast bathymetric file (.fbt) and faster navigation file (.fnv), and then mbm_plot is used to generate the colorful bathymetric map with tracklines, see figure1.

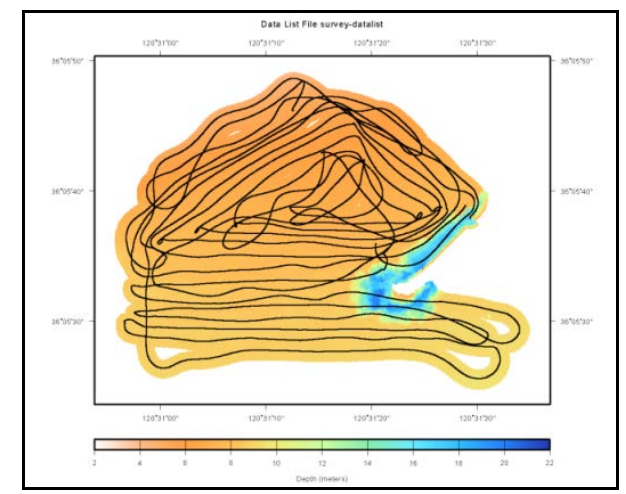

Figure1. The colorful bathymetric map with tracklines

Before the final measurement, the initial position of the transducer must be corrected, including roll, pitch, heading, and draft. Wherein the initial draft parameter can be measured after the installation. The other three parameters need to layout measuring lines, and are calculated by the correct module of the acquisition software ${ }^{[7]}$. Sound velocity correction includes two aspects: (1) the surface sound velocity, i.e. the sound velocity at the transducer. It can be real-timely acquired when installing surface sound velocity probe; (2) profile of sound velocity, real-timely acquired when installing sound velocity profiler ${ }^{[8]}$. As the water observation is the value relative to the instantaneous sea level and this program is required to be based on Qingdao Maidao tide station water zero, the tide must be corrected after the above mentioned corrections. The tide data is provided by Qingdao Maidao tide station.

If the raw data to correct the measure lines is exist, MB-System can also determine the roll 
parameter. Firstly, mbcopy is used to cut the data and only useful data is left. Then run mbprocess to process the data and finally use mbrollbias to calculate the roll parameter.

As the navigation methods are different under and above water, mbnavedit provides three correction methods: (1) Editing. This method is suitable to most cases, which just need to correct navigation data, i.e. to delete obvious wrong values and interpolation; (2) Modelling. This method is for the case that position value is invalid but course and speed are valid. Reasonable adaptive approximate navigation data can be obtained from Dead Reckoning navigation model; (3) Adjustment. This is for the navigation data is reliable but water depth is not consistent to sonar overlap characteristics. By adjusting navigation to match them. In this program, Reson7125 is fixed to the ship's side, and high precision positioning and inertial navigation system is adopted. Therefore, the navigation positioning data is reliable, and mbnavedit is enough for the first method to correct navigation data.

In MB-System, mbedit and mbclean are used to edit depth data. The former is based on some rules to fulfill automatic edition, and can be used to batch process. The latter can do interactive processing, but limited to one single line. Mbclean has many clean rules, like -X zap_beam means to delete the edge beams of zap_beams, -B low/high means to delete depth values outside of the range low to high, -C slope/unit means to delete depth value where the slope is larger than 'slope', unit is optional, default value is 0 . Following is ambclean command:

\begin{tabular}{|cc|}
\hline $\begin{array}{c}\text { \$mbclean }-F 121 \\
\text { 20141030-014638.mb121 -X5 }\end{array}$ & -I \\
\hline
\end{tabular}

This command is to delete 5 edge beams, the result can be seen with mbedit, as figure2. Dots marked as bule are deleted beams.

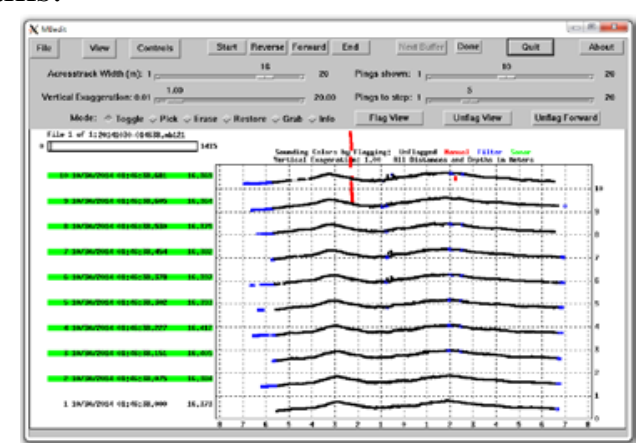

Fig2. Map of processedresult using mbclean

Before using mbprocess to precess measuring lines, MB-System already generated .par files for each line files. The parameter file contains all the information required for data processing, including navigation correction, roll, heading correction, draft, tide, sound velocity etc. These parameters can be assigned by mbset. The following command is used in the program to assign tide to all the lines.

\$mbset -F-1 -I datalist
-PTIDEMODE:1
-PTIDEFILE:20141225.tid
-PTIDEFORMAT:2

After setting parameters, mbprocess is used to process raw measuring lines, and then mbm_grid convert the processed lines to grid files, and finally mbgrdviz is used to view the grids, see figure3. 


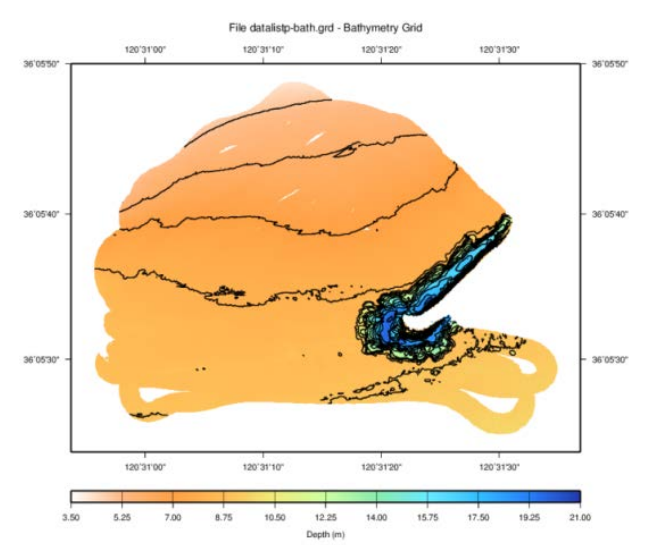

Fig3.the final result achieved by MB-System

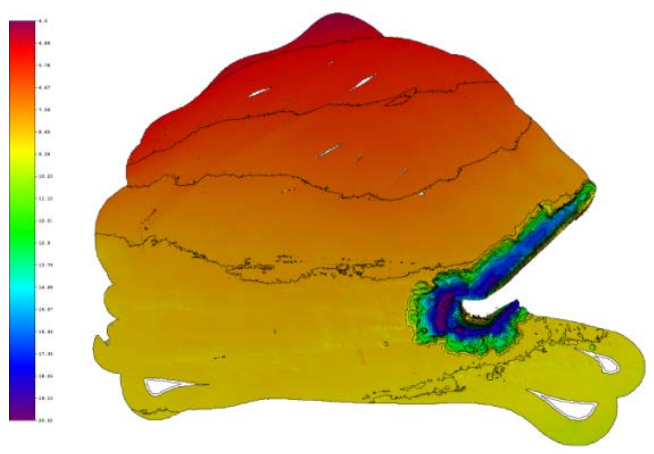

Fig4.the final result achieved by Caris

The process result using Caris software is shown as Figure4. Compared to Figure3, isobaths are in good agreement, and it can be seen that the result achieved by MB-System is reliable.

\section{Conclusion}

From the data process and the final result, it can be seen that processing multibeam data based on Cygwin and MB-System is feasible and reliable. With Cygwin, the use and study of MB-System can be done on Windows platform without the requirement of switching operation system. Compared to the commercial software, getting started with MB-System might be relatively difficult, but it make us close to the data and help us understand the principles of multibeam. Besides, the MB-Sytem is open source, which means that it can be not only directly used but also expanded, and it can be a great help to relate researchers.

\section{Acknowledgements}

This work was supported by the project of North China Sea Branch of State Oceanic Administration(Grant NO. 2015B18).

\section{References}

[1] Redhat Inc. Cygwin User's Guide [P/OL]. 2014. http: // www. cygwin. com/ cygwinugnet. html.

[2] David W. Caress, Dale N. Chayes. Seafloor Mapping Software:Processing and Display of Swath Sonar Data[EB/OL].2014.http://www.mbari.org/data/mbsystem/index.html

[3] Gao Bo,Fu Renqi.Multibeam Sounding Post-processing Techniques Based on MB-System[J]. Computer CD Software and Applications.Oct.2011:51 52 
[4] Hu Qinghua,Zhang Jianhua,et al.Reasearch on Using MB_System to Process Multibeam Bathymetric Data Based on Linux[J].Hydrographic Surveying and Charting.Vol.26,No.5,Sep.,2006:28-30

[5]xiaokcehui. Installing mbsystem5.3.2207 on Cygwin1.7.33-1[EB/OL] 2015(1). http://www.xiaokcehui.com/article/hcrj/20.html

[6]Generic Sensor format specification Vesion2.02 SAIC doc 982-15g[S] .20, June ,2003

[7] Liu Shengxuan,Guan Yongxian.Judgement and Correction of Parameter Error of Multibeam System [J]. Hydrographic Surveying and Charting. Vol.22, No.1, Jan., 2002:33-37

[8]Zhao Jianhu,Liu Jingnan. Multibeam Bathymetric and Image Data Processing [M] Wuhan: Wuhan University Press Sep.,2008

[9] Val Schmidt, Dale Chayes, Dave Caress. The MB-System cookbook[EB/OL].20,Feb.,2004 